\title{
MAGNETOROTATIONAL MECHANISM OF SUPERNOVA EXPLOSIONS - RESULTS OF 2D SIMULATIONS
}

\author{
S.G. MOISEENKO \\ Space Research Institute, Moscow, Russia \\ moiseenko@mx.iki.rssi.ru
}

Results of 2D numerical simulation of the magnetorotational mechanism of a supernova explosion are presented. Simulation has been done for the real equations of state and neutrino energy losses have been taken into account. Simulation has been done on the basis of an Implicit Lagrangian scheme on a triangular grid with grid reconstructuring. It is shown that, due to differential rotation of the star, a toroidal component of the magnetic field appears and grows with time. Rotational momentum transfers outwards as the toroidal component grows with time. With the evolution of the process, part of the envelope of the star is ejected. The amounts of the thrown-off mass and energy are estimated. The results of the simulation could be used as a possible explanation for the supernova explosion picture. 